\title{
MOLECULAR DOCKING ANALYSIS OF Azadirachta indica CONSTITUENTS AS INHIBITORS OF AFLATOXIN POLYKETIDE SYNTHASE (APKS)
}

\author{
Solomon Abrehame ${ }^{1,2}$, Merry Hailu ${ }^{1,3}$, Valsa Remony Manoj ${ }^{1}$, Yen-Po Chen ${ }^{4}$, \\ 5 , Radhakrishnan Narayanaswamy, ${ }^{6}$, \\ ${ }^{1}$ Department of Biotechnology, Vel Tech Rangarajan Dr.Sagunthala R \& D Institute of Science \\ and Technology, Avadi, Chennai-62, Tamil Nadu, India. \\ ${ }^{2,3}$ Veterinary Drug and Feed Administration and Control Authority of Ethiopia [VDFACA], ${ }^{2}$ \\ (Veterinary Drug Registration, Certification and Administration Directorate Director), \\ ${ }^{3}$ (Animal products, Veterinary Drug and Feed Quality Control Assessment Center, Toxicology \\ Analysis Team Leader), Addis Ababa, Ethiopia. \\ ${ }^{4,5}$ Department of Animal Science and ${ }^{5}$ The iEGG and Animal Biotechnology Center, National \\ Chung Hsing University, Taichung 40227, Taiwan. \\ ${ }^{6}$ Department of Biochemistry, St. Peter's Institute of Higher Education and Research (SPIHER), \\ Avadi, Chennai-54, Tamil Nadu, India. \\ ${ }^{\otimes}$ Corresponding Author: nrkishnan@gmail.com
}

\begin{abstract}
In the present study, 18 selected Azadirachta indica constituents, which includes azadiractin, azadiron, epicatechin, epoxyazadiradione, gallic acid, gedunin, isomargolonone, kaempferol, mahmoodin, margolone, margolonone, myristinin A, nimbolide, nimocinolide, quercetin, quercitrin, rutin and sugiol were assessed on the docking behaviour of Aflatoxin polyketide synthase (APKS) by utilizing PatchDock method. Furthermore, Molecular physico-chemical, Bioactivity scores and Absorption, Distribution, Metabolism and Excretion (ADME) analyses were also carried out using Molinspiration and Swiss ADME respectively. The molecular Physico-chemical analysis predicted that rutin has shown three violations for Lipinski's rule of five. And whereas, ADME analysis also showed to have high gastro-intestinal (GI) absorption effect for all the ligands (except for azadiractin, quercetin, quercitin and rutin). The docking studies revealed that myristinin A showed the best binding energy $(-350.92 \mathrm{kcal} / \mathrm{mol})$ for the target enzyme Aflatoxin polyketide synthase (APKS). Thus the present findings provide new insights in understanding 18 Azadirachta indica constituents as a possible inhibitor against PKS.
\end{abstract}

Keywords: Molecular Docking, Aflatoxin Polyketide Synthase (APKS), Azadiractin, Myristinin A, Isomargolonone.

RASĀYAN J. Chem., Vol. 14, No.2, 2021

\section{INTRODUCTION}

Food and animal feed contamination by mycotoxin is one of the causative factors responsible for food insecurity throughout the world. ${ }^{1}$ Aspergillus, Fusarium and Penicillium are the three genera of fungi which are generally reported to produce mycotoxins. ${ }^{2}$ Aflatoxins are one of the key mycotoxins produced by the Aspergillus species. Twenty different types of aflatoxins have been reported so far. Aflatoxins are highly carcinogenic, toxic and cause food contamination which in turn leads to various health issues. ${ }^{3}$ Aflatoxins have been reported to possess a serious impact on the social, political and economic aspects of society. ${ }^{4}$ Polyketides are the secondary metabolites produced by bacteria and fungi. Biogenesis of aflatoxin in Aspergillus flavus is a complex process and usually, polyketides are synthesis by a mega protein complex called polyketide synthase (PKS) with multiple domains. ${ }^{5}$

In particular, type I polyketide synthase belongs to fungi, which has been reported in many toxigenic Aspergillus species. ${ }^{6}$ Polyketide synthase (PKS) contains three major domains (like acyl carrier proteins,

Rasayan J. Chem., 14(2), 920-929(2021)

http://dx.doi.org/10.31788/ RJC.2021.1426063 
RASĀYAN J. Chem.

Vol. 14 | No. 2 |920-929| April - June | 2021

acyl transferase and ketoacyl synthase) and five other domains (like cyclase, dehydratase, enol reductase, keto reductase and methyl transferase). Moreover, inhibiting (or) modulating polyketide synthase (PKS) activity will reduce aflatoxin biosynthesis. ${ }^{7}$ Neoeriocitrin has been reported to inhibit the polyketide synthase. ${ }^{6}$ Similarly, five other natural compounds (such as astraglin, azadiradione, catechin, nimbin and salanin) have been reported to inhibit the polyketide synthase. ${ }^{5}$ The previous reports motivated us to perform the current study on 18 chosen Azadirachta indica constituents, which includes azadiractin, azadiron, epicatechin, epoxyazadiradione, gallic acid, gedunin, isomargolonone, kaemferol, mahmoodin, margolone, margolonone, myristinin $\mathrm{A}$, nembolide, nimocinolide, quercetin, quercetrin, rutin and sugiol. These 18 Azadirachta indica constituents were studied on the docking analysis of Aflatoxin polyketide synthase (APKS) by utilizing PatchDock method. Moreover, molecular Physico-chemical, bioactivity score and Absorption, Distribution, Metabolism and Excretion (ADME) analyses) were also performed using molinspiration and Swiss ADME respectively.

\section{EXPERIMENTAL}

\section{Ligand Preparation}

Chemical structures of selected ligands namely i) azadiractin [CID 5281303]; ii) azadiron [ CID 185587]; iii) epicatechin [CID 1203]; iv) epoxyazadiradione [CID 122801]; v) gallic acid [CID 370]; vi) gedunin [CID 12004512]; vii) isomargolonone [CID 189727]; viii) kaempferol [CID 5280863]; ix) mahmoodin [CID 126566]; x) margolone [CID 189728]; xi) margolonone [CID 189726]; xii) myristinin-A [CID 497359]; xiii) nimbolide [CID 100017], xiv) nimocinolide [CID 6442906]; xv) quercetin [CID 5280343]; xvi) quercitrin [CID 5280459]; xvii) rutin [CID 5280805] and xviii) sugiol [CID 94162] were downloaded from PubMed (www.PubMed.com) database. The energy minimized three dimensional chemical structures were performed for Patch Dock study. ${ }^{8-13}$

\section{Target Protein Preparation}

The 3D (three-dimensional) structure of the Aflatoxin polyketide synthase (PDB ID no: 3ILS with a resolution of $1.7 \AA$ ) was retrieved from the Protein Data Bank (www.rcsb.org). A chain of APKS protein was processed by removing all non-standard molecules including the crystallographically observed water particles (water without hydrogen bonds). The APKS protein was prepared/processed using UCSF Chimera software (www.cgi.ucsf.edu/chimera). ${ }^{8-9}$

\section{Molecular Physico-chemical and Bioactivity Score/Drug-likeness Analysis}

Molecular Physico-chemical and bioactivity score/drug-likeness analysis were also carried out using molinspiration online tool, according to the earlier report. ${ }^{8}$

\section{ADME Analysis}

Absorption, Distribution, Metabolism and Excretion (ADME) analysis was carried out using the Swiss ADME analysis method. ${ }^{8}$

\section{Docking Studies}

Docking studies were performed by the PatchDock online server (http://bioinfo3d.cs.tau.ac.il/PatchDock). PatchDock uses a geometry-based molecular docking algorithm method was utilized to recognize the binding scores and residues and also atomic contact energy (ACE) of the given ligands (18 Azadirachta indica constituents). Finally, the binding site analysis was done by using PyMOL software (www.pymol.org). ${ }^{8}$

\section{RESULTS AND DISCUSSION}

Azadirachta indica is a divine tree common name in English is "neem", which is native to Burma and India. ${ }^{14} A$. indica has been used in Indian traditional medicine since immemorial time. ${ }^{15}$ More than 100 chemical constituents have been reported from $A$. indica that include ascorbic acid, azadirachtin, azadirone, catechin, cerebroside, chlorogenic acid, cyclic trisulphide, cyclic tetrasulphide, deacetylnimbin, ellagic acid, epicatechin, epigallocatechin, ferulic acid, gallic acid, gedumin, nhexacosanol, hyperoside, isomargolonone, kaempferol, limonoids, lupeol, mahmoodin, margolone, margolonone, myricetin, myristinin A, nembolide, nimbandiol, nimbic acid, nimbidic acid, nimbidin, nimbiol, nimbolide, nimbolinin, protomeliacins, quercetin, quercetrin, rutin, salannin, scopoletin, $\beta-$ 
RASĀYAN J. Chem.

Vol. 14 | No. 2 |920-929| April - June | 2021

sitosterol, sodium nimbidinate, stigmasterol, valasinin and zafaral., ${ }^{5,16-19}$ Thus in the present study, 18 Azadirachta indica constituents were chosen as ligands as tabulated in the Table-1.

Table-1: Represents the Two-dimensional (2D) and Three-dimensional (3D) Structures of 18 Azadirachta indica Constituents

\begin{tabular}{|c|c|c|c|}
\hline S. No. & $\begin{array}{c}\text { Name of the Selected } \\
\text { Ligands (with Pubchem CID } \\
\text { no) }\end{array}$ & Two Dimensional (2D) Structures & $\begin{array}{c}\text { Three Dimensional (3D) } \\
\text { Structures }\end{array}$ \\
\hline 1. & $\begin{array}{c}\text { Azadiractin } \\
\text { [CID-5281303] }\end{array}$ & & \\
\hline 2. & $\begin{array}{c}\text { Azadiron } \\
\text { [CID-185587] }\end{array}$ & & \\
\hline 3. & $\begin{array}{l}\text { Epicatechin } \\
\text { [CID-1203] }\end{array}$ & & \\
\hline 4. & $\begin{array}{l}\text { Epoxyazadiradione } \\
\text { (CID-122801) }\end{array}$ & & \\
\hline 5. & $\begin{array}{l}\text { Gallic acid } \\
\text { [CID-370] }\end{array}$ & & \\
\hline
\end{tabular}


RASĀYAN J. Chem.

Vol. 14 | No. 2 |920-929| April - June | 2021

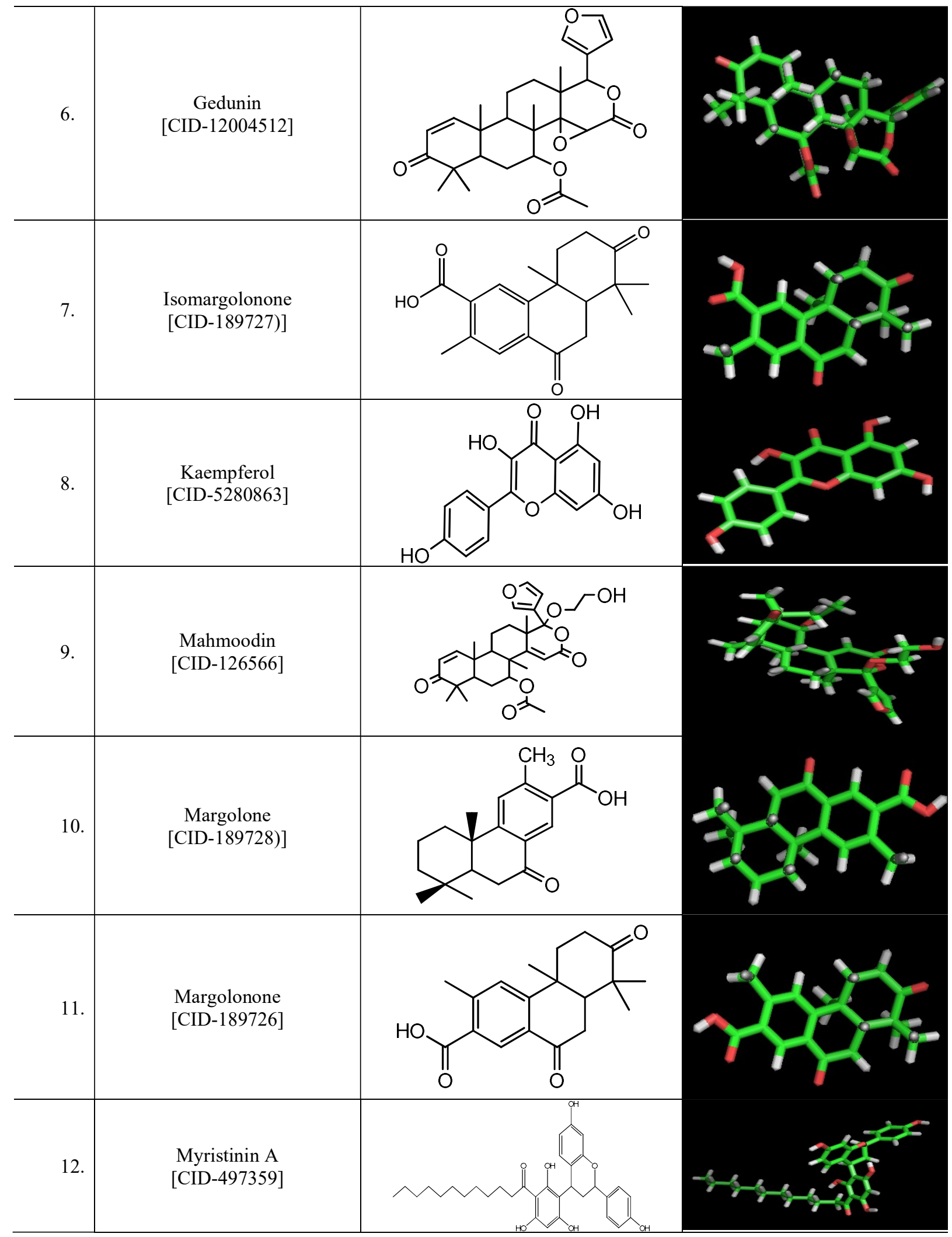


RASĀYAN J. Chem.

Vol. 14 | No. 2 |920-929| April - June | 2021

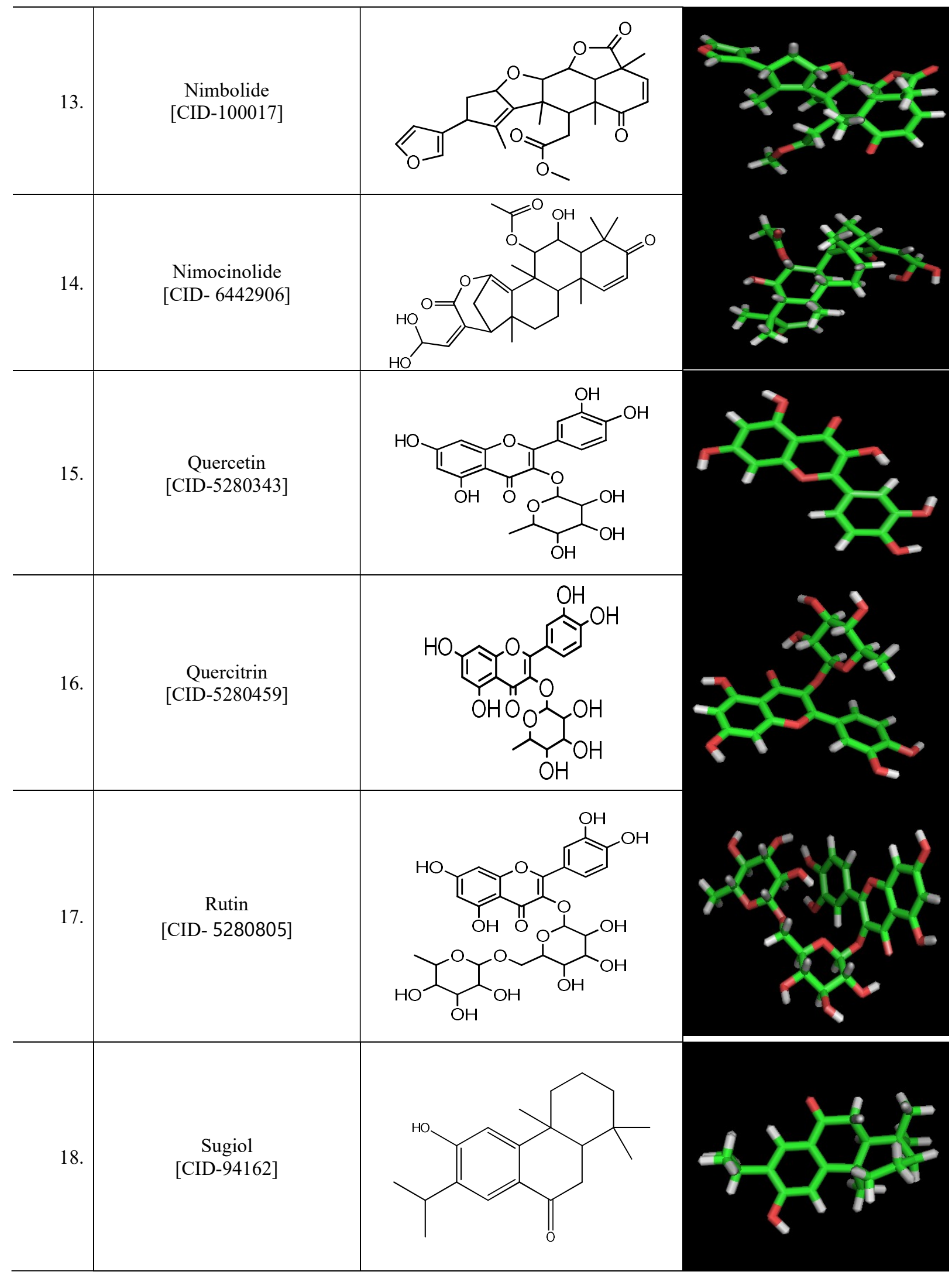


RASĀYAN J. Chem.

Vol. 14 | No. 2 |920-929| April - June | 2021

It is important to know the physio-chemical and bioactivity score of these 18 ligands (Azadirachta indica constituents) before carrying out the docking studies. Furthermore, Lipinski's rule of five (or) thumb of five was used to know the above-mentioned two properties and also aids in the analysis of the oral bioavailability of lead molecule. ${ }^{20}$ In general, in violation of the rule of five (RoF) is when-

(i) $\quad \log \mathrm{A}$ greater than 5 ;

(ii) Molecular Weight greater than 500;

(iii) Number of N, O (hydrogen bond receptor) greater than 10;

(iv) Number of $\mathrm{OH}$ and $\mathrm{NH}$ (hydrogen bond donor) greater than 5 and

(v) A number of the rotatable bond (rotb) greater than 15. However, in the present investigation, rutin has shown three violations, as shown the Table-2.

Similarly in the case of bioactivity score, if the score is greater than zero is "active", -5.0 to -0.0 is "moderately active" and less than -5.0 is "inactive." ${ }^{21}$ Except azadiractin and gallic acid, all other ligands showed "active" bioactivity score towards enzyme inhibitor descriptor as presented in Table-3. Similarly, ADME prediction is needed before performing docking analyses and this is generally agreed in the early stage of Drug - Discovery, Screening and Design, because of its special feature. ${ }^{22}$ Table-4 exhibited the ADME study of 18 ligands (Azadirachta indica constituents); except four ligands (azadiractin, quercetin, quercitin and rutin) all other ligands are shown to have high gastro-intestinal absorption property.

Table-2: Molecular Physico-chemical Descriptors Analysis of 18 ligands (Azadirachta indica constituents) using Molinspiration Online Software Tool

\begin{tabular}{|c|c|c|c|c|c|c|c|c|c|}
\hline Ligand Names & $\log A^{*}$ & TPSA $^{\circ}$ & Natoms ${ }^{\diamond}$ & MW: & $\mathrm{nON}$ & nOHNH & Nviolations & Nrotba & Volume $^{* *}$ \\
\hline Azadiractin & 1.42 & 215.37 & 51 & 720.72 & 16 & 3 & 2 & 10 & 611.69 \\
\hline Azadiron & 5.33 & 56.52 & 32 & 436.59 & 4 & 0 & 1 & 1 & 423.93 \\
\hline Epicatechin & 1.37 & 110.37 & 21 & 290.27 & 6 & 5 & 0 & 1 & 244.14 \\
\hline Epoxyazadiradione & 3.66 & 86.11 & 34 & 466.57 & 6 & 0 & 0 & 3 & 430.17 \\
\hline Gallic acid & 0.59 & 97.98 & 12 & 170.12 & 5 & 4 & 0 & 1 & 135.10 \\
\hline Gedunin & 4.34 & 95.35 & 35 & 482.57 & 7 & 0 & 0 & 3 & 439.15 \\
\hline Isomargolonone & 3.17 & 71.44 & 23 & 314.384 & 4 & 1 & 0 & 1 & 293.81 \\
\hline Kaempferol & 2.17 & 111.12 & 21 & 286.24 & 6 & 4 & 0 & 1 & 232.07 \\
\hline Margolonone & 3.17 & 71.44 & 23 & 314.38 & 4 & 1 & 0 & 1 & 293.81 \\
\hline Myristinin A & 8.58 & 127.44 & 40 & 548.68 & 7 & 5 & 2 & 13 & 519.11 \\
\hline Nimbolide & 1.94 & 92.06 & 34 & 466.53 & 7 & 0 & 0 & 4 & 417.03 \\
\hline Nimocinolide & 3.43 & 130.37 & 36 & 500.59 & 8 & 3 & 1 & 3 & 456.40 \\
\hline Quercetin & 0.64 & 190.28 & 32 & 448.38 & 11 & 7 & 2 & 3 & 363.95 \\
\hline Quercitrin & 0.64 & 190.28 & 32 & 448.38 & 11 & 7 & 2 & 3 & 363.95 \\
\hline Rutin & -1.06 & 269.43 & 43 & 610.52 & 16 & 10 & 3 & 6 & 496.07 \\
\hline Sugiol & 5.51 & 37.30 & 22 & 300.44 & 2 & 1 & 1 & 1 & 306.04 \\
\hline
\end{tabular}

"Octanol-Water partition coefficient, ${ }^{*}$ Polar surface area, ${ }^{\diamond}$ Number of non-hydrogen atoms, $\bullet$ Molecular weight, $\square$ Number of hydrogen bond acceptors [ $\mathrm{O}$ and $\mathrm{N}$ atoms], Number of hydrogen bond donors [ $\mathrm{OH}$ and $\mathrm{NH}$ groups], Number of Rule of 5 violations, ${ }^{\circ}$ Number of rotatable bonds, ${ }^{* *}$ Molecular volume.

Table-3: Drug-likeness Property Analysis of 18 Ligands (Azadirachta indica constituents) using Molinspiration Tool

\begin{tabular}{c|c|c|c|c|c|c}
\hline Ligand names & $\begin{array}{c}\text { GPCR** } \\
\text { ligand }\end{array}$ & $\begin{array}{c}\text { Ion channel } \\
\text { modulator } \\
\text { (ICM) }\end{array}$ & $\begin{array}{c}\text { Kinase } \\
\text { inhibitor } \\
(\mathrm{KI})\end{array}$ & $\begin{array}{c}\text { Nuclear } \\
\text { receptor } \\
\text { ligand (NRL) }\end{array}$ & $\begin{array}{c}\text { Protease } \\
\text { inhibitor } \\
(\mathrm{PI})\end{array}$ & $\begin{array}{c}\text { Enzyme } \\
\text { inhibitor } \\
(\mathrm{EI})\end{array}$ \\
\hline Azadiractin & -0.71 & -1.51 & -1.46 & -0.67 & -0.35 & -0.71 \\
\hline Azadiron & 0.13 & 0.11 & -0.54 & 0.47 & 0.06 & 0.44 \\
\hline Epicatechin & 0.41 & 0.14 & 0.09 & 0.60 & 0.26 & 0.47 \\
\hline Epoxyazadiradione & 0.18 & 0.17 & -0.40 & 0.60 & 0.16 & 0.47 \\
\hline Gallic acid & -0.77 & -0.26 & -0.88 & -0.52 & -0.94 & -0.17 \\
\hline Gedunin & 0.20 & 0.02 & -0.46 & 0.55 & 0.11 & 0.42 \\
\hline Isomargolonone & 0.24 & 0.02 & -0.46 & 0.69 & -0.19 & 0.36 \\
\hline Kaempferol & -0.10 & -0.21 & 0.21 & 0.32 & -0.27 & 0.26 \\
\hline
\end{tabular}


RASĀYAN J. Chem.

Vol. 14 | No. 2 |920-929| April - June | 2021

\begin{tabular}{c|c|c|c|c|c|c}
\hline Margolonone & 0.24 & 0.02 & -0.46 & 0.69 & -0.19 & 0.36 \\
\hline Myristinin A & 0.07 & -0.30 & -0.25 & 0.17 & -0.01 & 0.12 \\
\hline Nimbolide & 0.22 & 0.20 & -0.36 & 0.32 & 0.04 & 0.36 \\
\hline Nimocinolide & 0.14 & 0.14 & -0.40 & 0.63 & 0.24 & 0.74 \\
\hline Quercetin & -0.01 & -0.08 & 0.08 & 0.17 & -0.06 & 0.37 \\
\hline Quercitrin & -0.01 & -0.08 & 0.08 & 0.17 & -0.06 & 0.37 \\
\hline Rutin & -0.05 & -0.52 & -0.14 & -0.23 & -0.07 & 0.12 \\
\hline Sugiol & 0.30 & 0.19 & -0.24 & 0.71 & -0.23 & 0.38 \\
\hline
\end{tabular}

**GPCR- G Protein coupled receptors

Table-4: ADME Analyses of 18 Ligands (Azadirachta indica constituents) using Swiss ADME Online Tool

\begin{tabular}{c|c|c|c|c|c|c|c|c|c}
\hline Ligand Names & GI & BBB & P-gp & $\bullet$ CP1A2 & CYP2C19 & $\begin{array}{c}\bullet \text { CYP2 } \\
\text { C9 }\end{array}$ & $\begin{array}{c}\text { CYP2 } \\
\text { D6 }\end{array}$ & $\begin{array}{c}\bullet \text { CY3 } \\
\text { A4 }\end{array}$ & $\begin{array}{c}\text { Log } \mathrm{K}_{\mathrm{p}} \\
(\mathrm{cm} / \mathrm{s})\end{array}$ \\
\hline Azadiractin & Low & No & Yes & No & No & No & No & No & -9.92 \\
\hline Azadiron & High & No & No & No & No & No & No & No & $-4,90$ \\
\hline Epicatechin & High & No & Yes & No & No & No & No & No & -7.82 \\
\hline $\begin{array}{c}\text { Epoxyazadirad } \\
\text { ione }\end{array}$ & High & No & Yes & No & No & No & No & No & -6.14 \\
\hline $\begin{array}{c}\text { Gallic acid } \\
\text { Gedunin }\end{array}$ & High & No & No & No & No & No & No & No & -6.84 \\
\hline $\begin{array}{c}\text { Isomargolono } \\
\text { ne }\end{array}$ & High & Yes & Yes & No & Yes & No & No & Yes & -6.17 \\
\hline Kaempferol & High & No & No & Yes & No & No & Yes & Yes & -6.70 \\
\hline Margolonone & High & Yes & Yes & No & Yes & No & No & Yes & -6.17 \\
\hline Myristinin A & Low & No & Yes & No & No & No & No & No & -3.33 \\
\hline Nimbolide & High & No & Yes & No & No & No & No & No & -7.61 \\
\hline Nimocinolide & High & No & Yes & No & No & No & No & No & -7.75 \\
\hline Quercetin & Low & No & No & No & No & No & No & No & -8.42 \\
\hline Quercitrin & Low & No & No & No & No & No & No & No & -8.42 \\
\hline Rutin & Low & No & Yes & No & No & No & No & No & -10.26 \\
\hline Sugiol & High & Yes & No & No & Yes & Yes & No & No & -4.14 \\
\hline
\end{tabular}

"-Gastrointestinal (GI) absorption, "-Blood-brain barrier (BBB) permeant, "-P-gp-(P-glycoprotein) substrate, " Cytochrome (CYP) P450 Inhibitors, ${ }^{\circ}$-Skin Permeation (in $\mathrm{cm} / \mathrm{s}$ ).

Polyketides (natural products) are usually biosynthetic in bacteria, fungi, plants and viruses by an enzyme called polyketide synthases. ${ }^{23,7}$ Polyketides acts as an important drug target against aflatoxin contamination. ${ }^{24}$ However, polyketide synthases (PKS) have not been utilized as (protein) drug targets against any microbial pathogen so far. ${ }^{23}$ Fungal polyketide synthases (type I) comprises important functional domains such as- (i) ketosynthase; (ii) acyltransferase; (iii) acyl carrier protein, as well as (iv) product template (PT) domain that regulates the aldol cyclization of poly-beta-ketone intermediates. ${ }^{25}$

Thus in the present study Aflatoxin polyketide synthase (APKS) was chosen as the target protein, where the docking studies and binding free energy calculations showed myristinin A has the maximum binding energy $(-350.92 \mathrm{kcal} / \mathrm{mol})$ with that of APKS as shown in Table-5.

Isomargolonone has exhibited the lowest binding energy $(-98.83 \mathrm{kcal} / \mathrm{mol})$ with that of APKS, whereas remaining Azadirachta indica constituents exhibited the following trend as shown below: kaempferol ($242.53 \mathrm{kcal} / \mathrm{mol})<$ quercetin $(-236.98 \mathrm{kcal} / \mathrm{mol})<$ epicatechin $(-234.27 \mathrm{kcal} / \mathrm{mol})<$ rutin $(-216.06$ $\mathrm{kcal} / \mathrm{mol})<$ azadiron $(-198.35 \mathrm{kcal} / \mathrm{mol})<$ gedunin $(-173.38 \mathrm{kcal} / \mathrm{mol})<\operatorname{margolonone}(-168.93 \mathrm{kcal} / \mathrm{mol})$ $<$ epoxyazadiradione $(-159.08 \mathrm{kcal} / \mathrm{mol})<\operatorname{mahmoodin}(-156.08 \mathrm{kcal} / \mathrm{mol})<$ nimocinolide $(-144.59$ 
RASĀYAN J. Chem.

Vol. 14 | No. 2 |920-929| April - June | 2021

$\mathrm{kcal} / \mathrm{mol})<$ nimbolide $(-140.58 \mathrm{kcal} / \mathrm{mol})<\operatorname{sugiol}(-137.63 \mathrm{kcal} / \mathrm{mol})<$ quercitrin $(-134.37 \mathrm{kcal} / \mathrm{mol})<$ margolone $(-109.01 \mathrm{kcal} / \mathrm{mol})<$ gallic acid $(-108.92 \mathrm{kcal} / \mathrm{mol})$ and azadiractin $(-107.54 \mathrm{kcal} / \mathrm{mol})$. Interestingly epicatechin, gallic acid and kaempferol exhibited interaction with His2055 amino acid (AA) residue of APKS as shown in Table-5. The current finding showed in good agreement with the earlier report, namely five Azadirachta indica constituents such as astraglin, azadiradione, catechin, nimbin and salanin have been reported to inhibit the polyketide synthase activity. ${ }^{5}$

Table-5: Binding Energy Analyses of 18 Ligands (Azadirachta indica constituents) with that of Aflatoxin Polyketide

\begin{tabular}{|c|c|c|c|c|}
\hline S. No. & Ligand Names & $\begin{array}{c}-\mathrm{ACE} \\
(-\mathrm{kcal} / \mathrm{mol})\end{array}$ & $\begin{array}{l}\text { Interaction of Amino Acid } \\
\text { (AA) Residue }\end{array}$ & Bond Distance $(\AA)$ \\
\hline \multirow[t]{3}{*}{1.} & \multirow[t]{3}{*}{ Azadiractin } & \multirow[t]{3}{*}{107.54} & His1911 & 2.8 \\
\hline & & & Lys2021 & 3.4 \\
\hline & & & Phe2056 & 3.0 \\
\hline 2. & Azadiron & 198.35 & No & - \\
\hline \multirow[t]{2}{*}{3.} & \multirow[t]{2}{*}{ Epicatechin } & \multirow[t]{2}{*}{234.27} & His2055 & 2.6 \\
\hline & & & His2088 & 3.2 \\
\hline \multirow[t]{5}{*}{4.} & \multirow[t]{5}{*}{ Epoxyazadiradione } & \multirow[t]{5}{*}{159.08} & Ile1967 & 3.3 \\
\hline & & & Gln1969 & 3.2 \\
\hline & & & Arg2061 & 3.3 \\
\hline & & & Thr2062 & 2.6 \\
\hline & & & Glu2063 & 3.1 \\
\hline \multirow[t]{2}{*}{5.} & \multirow[t]{2}{*}{ Gallic Acid } & \multirow[t]{2}{*}{108.92} & Tyr1979 & 2.9 \\
\hline & & & His2055 & 3.0 \\
\hline \multirow[t]{2}{*}{6.} & \multirow[t]{2}{*}{ Gedunin } & \multirow[t]{2}{*}{173.38} & Gln1969 & 2.7 and 3.4 \\
\hline & & & Thr2062 & 3.6 \\
\hline 7. & Isomargolonone & 98.83 & Met2054 & 2.0 \\
\hline \multirow[t]{3}{*}{8.} & \multirow[t]{3}{*}{ Kaempferol } & \multirow[t]{3}{*}{242.53} & $\mathrm{G} \ln 1969$ & 3.6 \\
\hline & & & Tyr1979 & 3.2 \\
\hline & & & His 2055 & 3.3 \\
\hline 9. & Mahmoodin & 156.08 & No & - \\
\hline 10. & Margolone & 109.01 & No & - \\
\hline 11. & Margolonone & 168.93 & Glu2045 & 1.7 \\
\hline \multirow[t]{4}{*}{12 . } & \multirow[t]{4}{*}{ Myristinin A } & \multirow[t]{4}{*}{350.92} & $\mathrm{G} \ln 1969$ & 2.0 \\
\hline & & & Ala2038 & 2.3 \\
\hline & & & Phe2056 & 2.7 \\
\hline & & & Arg2061 & 3.2 \\
\hline 13. & Nimbolide & 140.58 & Thr2062 & 3.2 \\
\hline \multirow[t]{3}{*}{14.} & \multirow[t]{3}{*}{ Nimocinolide } & \multirow[t]{3}{*}{144.59} & Met2054 & 2.4 \\
\hline & & & Phe2056 & 2.4 \\
\hline & & & Thr2062 & 3.5 \\
\hline 15. & Quercetin & 236.98 & Tyr1979 & 3.0 \\
\hline \multirow[t]{5}{*}{16.} & \multirow[t]{5}{*}{ Quercitrin } & \multirow[t]{5}{*}{134.37} & Gln1969 & 2.8 and 3.3 \\
\hline & & & $\mathrm{G} \ln 2059$ & 2.4 \\
\hline & & & Lys2060 & 2.2 \\
\hline & & & Thr2062 & 3.6 \\
\hline & & & Asp2067 & 2.4 \\
\hline 17. & Rutin & 216.06 & Glu1916 & 2.2 \\
\hline & & & Asn 1920 & 3.0 \\
\hline 18. & Sugiol & 137.63 & Arg2061 & 3.5 \\
\hline
\end{tabular}

- - Atomic Contact Energy (ACE)

\section{CONCLUSION}

In the present investigation, entire 18 constituents/ligands from Azadirachta indica have exhibited the potential to bind and dock with Aflatoxin polyketide synthase (APKS) except for three ligands (azadiron, 
RASĀYAN J. Chem.

Vol. 14 | No. 2 |920-929| April - June | 2021

mahmoodin and margolone). Isomargolonone has exhibited the lowest binding energy with that of PKS. Thus, it is firmly proposed that the findings of the current study may give new knowledge about these 18 ligands (Azadirachta indica constituents) as potential APKS inhibitors concerning the prevention of aflatoxin contaminations.

\section{ACKNOWLEDGMENT}

The first three authors (SA, MH and VRM) acknowledge Vel Tech Rangarajan Dr. Sagunthala R \& D Institute of Science and Technology [Deemed to be University], Chennai for the support provided towards the successful completion of this research work. The author Yen-Po Chen acknowledges the financial support by The iEGG and Animal Biotechnology (AB) Center from The Feature Area Research Center Program (within the framework of the Higher Education Sprout Project) by the Ministry of Education $[\mathrm{MOE}]$ in Taiwan.

\section{REFERENCES}

1. P. Udomkun, A. N. Wiredu, M. Nagle, J. Müller, B. Vanlauwe and R. Bandyopadhyay, Food Control, 76(3), 127(2017), DOI:10.1016/j.foodcont.2017.01.008

2. K. R. N. Reddy, B. Salleh, B. Saad, H. K. Abbas, C. A. Abel and W. T. Shier, Toxin Reviews, 29(1), 3(2010), DOI: 10.3109/15569541003598553

3. D. K. Mahato, K. E. Lee, M. Kamle, S. Devi, K. N. Dewangan, P. Kumar and S. G. Kang, Frontiers in Microbiology, 10, 2266(2019), DOI:10.3389/fmicb.2019.02266

4. M. K. Pandey, R. Kumar, A. K. Pandey, P. Soni, S. S. Gangurde, H. K. Sudini, J. C. Fountain, B. Liao, H. Desmae, P. Okori and X. Chen, Toxins, 11(6), 315(2019), DOI:10.3390/toxins1 1060315

5. G. Lavanya and D. Vanila, International Journal of Pharmaceutical and Clinical Research, 7(1), 5(2015).

6. M. Kumar, G. Sahoo, R. Topno, K. Pandey, V. Ravidas, M. Dikhit, H. Kumar and P. Das, International Journal of Infectious Diseases, 73, 285(2018), DOI:10.1016/j.jijid.2018.04.4065

7. S. Tiwari, S. K. Shishodia and J. Shankar, 3 Biotech, 9(4), 149(2019), DOI:10.1007/s13205-019$1675-y$

8. V. Vijayakumar, N. Radhakrishnan, C. Rameshkumar, IEEE ICSTM, 513(2017), DOI: 10.1109/ICSTM.2017.8089213

9. N. Radhakrishnan, K. W. Lam and I. S. Intan, International Journal of Food Properties, 18(10), 2155(2015), DOI: 10.1080/10942912.2014.963870

10. M. Muchtaridi , S. Megantara, D. Dermawan and M. Yusuf, Rasayan Journal of Chemistry, 12(4), 1927(2019), DOI: 10.31788/RJC.2019.1245391

11. B. Geetha, G. Swarnalatha and G. V. Subba Reddy, Rasayan Journal of Chemistry, 12(3), 1063(2019), DOI: 10.31788/RJC.2019.1235165a

12. M. Malathi and D. Prabhakarachary, Rasayan Journal of Chemistry, 12(3), 1347(2019), DOI: $10.31788 /$ RJC.2019.1235290

13. I. Irfan, M. Irfan, M. Abid and A. Azam. Rasayan Journal of Chemistry, 12(4), 2082(2019), DOI: $10.31788 /$ RJC.2019.1245320

14. T. Khogare Balaji and R. Kulal Shivaji, International Journal of Life Sciences, A10, 181(2018).

15. V. S. Kumar and V. Navaratnam, Asian Pacific Journal of Tropical Biomedicine, 3(7), 505(2013), DOI: $10.1016 / \mathrm{S} 2221-1691(13) 60105-7$

16. X. Luo, Y. Ma, S. Wu and D. Wu, Journal of Natural Products, 62(7), 1022(1999), DOI: $10.1021 / \mathrm{np} 980452 \mathrm{~d}$

17. K. Biswas, I. Chattopadhyay, R. K. Banerjee and U. Bandyopadhyay, Current Science, 82(11), 1336(2002).

18. D. K. Yadav, Y. P. Bharitkar, K. Chatterjee, M. Ghosh, N. B. Mondal and S. Swarnakar, Indian Journal of Experimental Biology, 54, 708(2016).

19. S. Saleem, G. Muhammad, M. A. Hussain and S. N. A. Bukhari, Phytotherapy Research, 32(7), 1241 (2018), DOI:10.1002/ptr.6076

20. C. A. Lipinski, F. Lambardo, B. W. Dominy and P. J. Feeney, Advanced Drug Delivery Reviews, 23 (1-3), 3(1997), DOI: 10.1016/S0169-409X(96)00423-1 
RASĀYAN J. Chem.

Vol. 14 | No. 2 |920-929| April - June | 2021

21. S. Singh, A. K. Gupta and A. Verma. Research Journal of Pharmaceutical, Biological and Chemical Science, 4(2), 876(2013).

22. J. Wang and L. Urban. Drug Discovery World, 5, 73 (2004).

23. A. Aggarwal, M. K. Parai, N. Shetty, D. Wallis, L. Woolhiser, C. Hastings, N. K. Dutta, S. Galaviz, R. C. Dhakal, R. Shrestha and S. Wakabayashi, Cell, 170(2), 249(2017), DOI: $10.1016 /$ j.cell.2017.06.025

24. A. G. Newman, A. L. Vagstad, K. Belecki, J. R. Scheerer and C. A. Townsend, Chemical Communications, 48, 11772(2012), DOI: 10.1039/c2cc36010a

25. J. F. Barajas, G. Shakya, G. Moreno, H. Rivera, D. R. Jackson, C. L. Topper, A. L. Vagstad, J. J. La Clair, C. A. Townsend, M. D. Burkart and S. C. Tsai, Proceedings of the National Academy of Sciences, 114 (21), E4142(2017), DOI:10.1073/pnas.1609001114

[RJC-6063/2020] 